\title{
Cellular Modeling of Cancer Therapy Induced Cardiotoxicity
}

\author{
William P Bozza, Keisha Melodi McSweeney and Baolin Zhang* \\ Center for Drug Evaluation and Research, Food and Drug Administration, USA
}

Submission: December 28, 2017; Published: January 08, 2018

"Correspondence Address: Dr. Baolin Zhang, Office of Biotechnology Products, Center for Drug Evaluation and Research, Food and Drug Administration, Silver Spring, MD 20993, Maryland, USA, Tel: 240-402-6740; Email: Baolin.zhang@fda.hhs.gov

Keywords: Human induced pluripotent stem cells; Cancer therapy; Cardiotoxicity; Cellular model; Biomarker; Cardiomyocytes

\section{Mini Review}

Drug-induced cardiotoxicity is a major safety concern in cancer therapy. Cytotoxic agents and targeted therapies used to treat cancer, including classic chemotherapeutic agents, monoclonal antibodies that target tyrosine kinase receptors, small molecule tyrosine kinase inhibitors, and even antiangiogenic drugs, all affect the cardiovascular system. Examples of clinical cardiotoxic side effects include reduction of left ventricular ejection fraction, arrhythmia, cardiomyopathy, myocarditis, heart failure, and even cardiac death [1,2]. The current clinical surveillance and monitoring approaches for cardiac dysfunction during cancer treatment provides limited patient benefit especially to those who have asymptomatic cardiac dysfunction. There is an unmet need for the identification of biomarkers that can pinpoint increased patient risk to drug induced cardiotoxicity before initiation of drug treatment.

During drug development, preclinical animal models are heavily employed to assess drug-induced cardiotoxicity $[3,4]$. However, animal models have several limitations. These models may not accurately recapitulate drug-induced cardiotoxicity due to species-specific differences in both drug metabolism and cardiac structure and function. Additionally, significant differences between mouse and human cardiac system in terms of electrophysiology and contractility features limit human extrapolation from findings [5,6]. Recently, human induced pluripotent stem cell-derived cardiomyocytes (hiPSC-CMs) have emerged as a powerful tool to model cardiotoxicity [7-9]. Pluripotent stem cells can be successfully differentiated into cardiomyocytes using in vitro techniques (e.g., embryoid body (EB) formation, monolayer culture, and inductive coculture) that recapitulate stepwise embryonic development $[10,11]$. Consistency in production is achieved through the tight control of differentiation media (e.g., growth factors and specific media components) coupled with validated isolation procedures using a combination of physical, genetic, and cell surface marker selection. Confirmatory characterization of isolated cardiomyocytes largely relies on monitoring changes in gene expression (cardiac transcription factors, myofilament proteins, and $\mathrm{Ca} 2+$ cycling proteins), detecting myofilament proteins by immunofluorescence microscopy, and measuring electrophysiological properties.

HiPSC-CM based assays are attractive cellular models of drug-induced cardiotoxicity due to their physiological relevance and their suitability for simplified assay development. hiPSC-CMs are species specific and maintain many of the properties of the human heart including beating dynamics, electrophysiological characteristics, and protein expression profiles. Additionally, since this is a cell based model, hiPSC-CMs are compatible with high throughput screening modalities allowing a large-scale assessment of multiple drugs and drug combinations. Moreover, new drug combinations can be tested for cardiotoxic risk using hiPSC-CMs. In principle, this model can be used universally to assess any drug product's potential to induce cardiotoxicity.

Recent work in our lab and in others has applied hiPSCCMs for studying drug induced cardiotoxicity. Among a variety of anticancer drugs, anthracyclines (e.g., doxorubicin) and the monoclonal antibody, trastuzumab, are agents with a wellknown cardiotoxicity [12]. Therefore, these agents and their combination(s) are widely used in relevant hiPSC-CM studies. Doxorubicin treated hiPSC-CMs displayed dose-dependent increases in cell death, reactive oxygen species (ROS), intracellular calcium concentration, mitochondria dysfunction, alterations in contractility measurements, and abnormalities in electrophysiological properties $[9,13]$. These phenotypic changes and biochemical responses agree well with previous 
in vivo findings $[14,15]$ showcasing the most applicable use of hiPSC-CMs as a preclinical model to screen compounds or drug candidates for their potential cardiac risk during drug development.

HiPSC-CMs can improve mechanistic understanding of drug-induced cardiotoxicity. For example, hiPSC-CMs have been successfully applied to demonstrate the mechanisms of doxorubicin induced cardiotoxicity including reactive oxygen species (ROS) generation [16], topoisomerase inhibition $[17,18]$, and alterations in calcium transport [19]. Recently hiPSC-CMs were utilized to confirm the involvement of topoisomerase II-B (TOP2B) in doxorubicin-induced cardiotoxicity. Specifically, genetic disruption of TOP2B gene in hiPSC-CMs led to a significant reduction in doxorubicin induced cell death [9]. Using hiPSC-CM assays, our lab has made a novel finding in that anthracyclines can induce cardiotoxicity through upregulation of surface death receptor proteins [13]. Moreover, apoptosis of hiPSC-CMs induced by doxorubicin was further enhanced by death ligands such as TNF-related apoptosis inducing ligand (TRAIL).

In a global context, when hiPSC-CM disease modeling is coupled with next-generation sequencing (NGS), an unbiased approach to understanding disease mechanisms can be utilized to discover previously unknown pathogenic mechanisms of cardiotoxicity. Gene expression profiling has been used to verify predicted mechanisms involved in the development of cardiotoxicity in response to doxorubicin [9,20]. Importantly, these studies have also identified other pathways, including genes involved in structural regulation of the heart and the development of cardiomyopathy [9]. Additionally, disease modeling using hiPSC-CM and NGS has also highlighted important temporal changes in global gene expression that mimic the human response to treatment and underscore the importance of early detection of cardiotoxicity. Acute response to low doses of anthracyclines has been shown to be reversible in comparison to repeated or chronic exposure which leads to irreversible cardiomyocyte damage [20]. Together these efforts demonstrate the power of a clinically relevant cell based model in driving new mechanistic discoveries of drug-induced cardiotoxicities.

HiPSC-CMs can also be used to identify clinically relevant biomarkers. Recent work has demonstrated a dose-dependent secretion of cTNI, FABP3, and NT-proBNP in cell culture media from hiPSC-CMs treated with known cardiotoxic drugs [21]. These data agree with clinical studies that support troponins and natriuretic peptides as biomarkers of drug-induced cardiotoxicity [22-24]. In a separate study, a metabolite signature of doxorubicin induced toxicity was identified by an HNMR screen of secreted metabolites in media from treated hiPSC-CMs [25]. This signature proposes acetate, formate, and pyruvate as candidate biomarkers of doxorubicin-induced cardiotoxicity [25]. However, these biomarkers are released only after damage to cardiomyocytes occur. Based on our laboratory's finding that anthracycline treatment can upregulate surface death receptors on cardiomyocytes, we propose that patients who are associated with elevated serum levels of circulating death ligands may be at higher risk to anthracycline-induced cardiotoxicity (Figure 1). Additional studies are underway in our lab through collaboration with a clinical institution to identify serum biomarkers that may help predict cardiac risks in individual patients prior to initiation of anthracycline treatment.

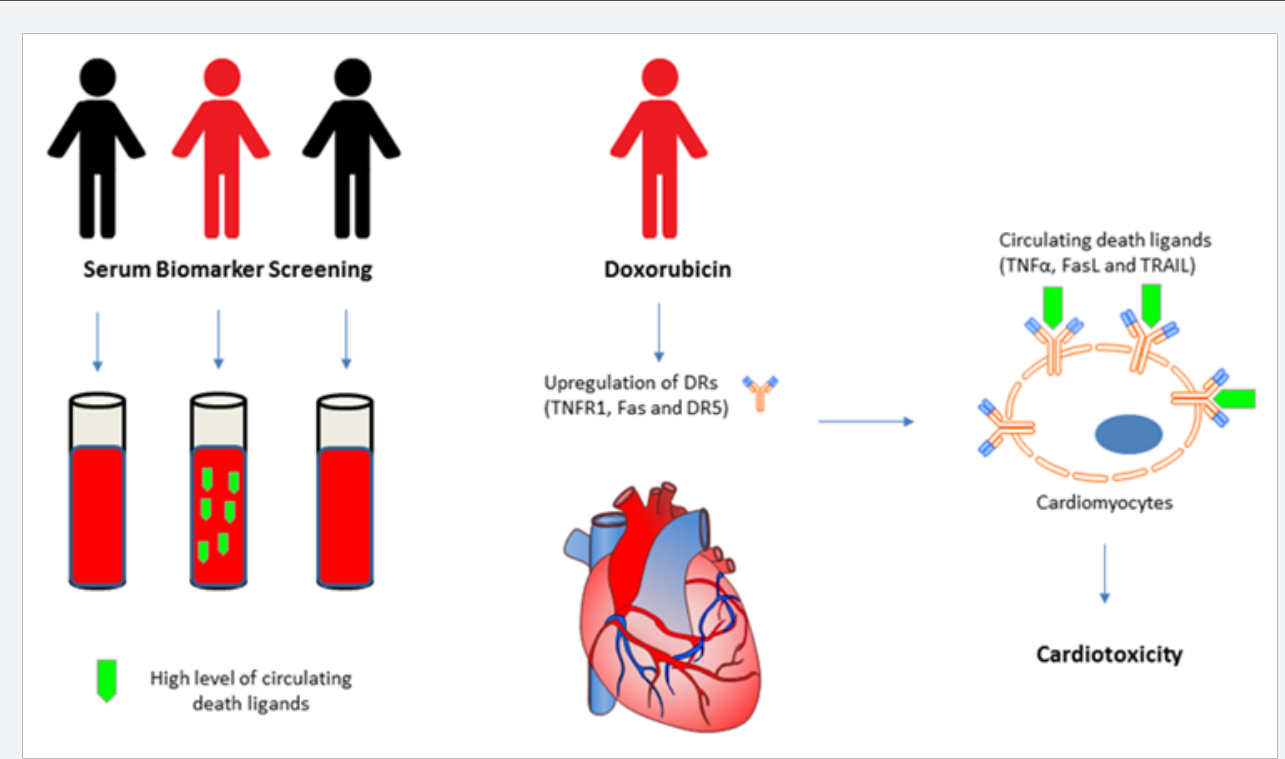

Figure 1: Identification of serum biomarkers to predict drug-induced cardiotoxicity. Our recent work demonstrates that anthracycline agents are potent inducers of death receptors in cardiomyocytes [13]. Studies are underway in our lab to evaluate the relationship between baseline serum levels of TNF-related death ligands and cardiac events in cancer patients undergoing anthracycline treatment. 
HiPSC-CMs obtained from commercial sources are typically derived from a single healthy donor who may not be representative of patient-specific cardiomyocytes. There is a growing interest to generate patient-specific hiPSC-CMs for gaining insights into drug-induced cardiotoxicity in individual patients. In this regard, patient specific hiPSC-CMs were shown to recapitulate clinically observed doxorubicin induced cardiotoxicity (DIC) [26]. Cardiomyocytes derived from breast cancer patients who experienced DIC were more sensitive to doxorubicin toxicity than those derived from breast cancer patients who did not experience DIC. The results from this work suggested lower basal metabolism and lower mitochondrial content of hiPSC-CMs could be key patient specific contributors in DIC [26].

Despite the promising applications of hiPSC-CMs, precautions should be taken when developing relevant assays to ensure the validity of the testing results. Currently there are several commercial sources that provide hiPSC-CMs. However, due to differences in differentiation and isolation protocols, hiPSC-CMs from different manufacturers may have differences in electrophysiological and phenotypic characteristics. Even from a single manufacturer batch-to-batch variability in hiPSCCM characteristics may exist. Nonetheless, when adequately characterized hiPSC-CMs can be used to model drug induced cardiotoxicity, increase mechanistic understanding of druginduced cardiotoxicity, and can potentially allow for the identification of novel cardiac safety biomarkers collectively contributing to improved cancer therapy.

\section{Conflict of Interest}

No conflicts of interest are disclosed.

\section{References}

1. Johnson DB, Justin M Balko, Margaret L Compton, S Chalkias, Joshua Gorham, et al. (2016) Fulminant Myocarditis with Combination Immune Checkpoint Blockade. N Engl J Med 375(18): 1749-1755.

2. Curigliano G, Cardinale D, Dent S, Criscitiello C, Aseyev O, et al. (2016) Cardiotoxicity of anticancer treatments: Epidemiology, detection, and management. CA Cancer J Clin 66(4): 309-325.

3. Houser SR, Margulies KB, Murphy AM, Spinale FG, Francis GS, et al. (2012) Animal models of heart failure: a scientific statement from the American Heart Association. Circ Res 111(1): 131-150.

4. Robert J (2007) Long-term and short-term models for studying anthracycline cardiotoxicity and protectors. Cardiovasc Toxicol 7(2): 135-139.

5. Clark WA Jr, Chizzonite RA, Everett AW, Rabinowitz M, Zak R (1982) Species correlations between cardiac isomyosins. A comparison of electrophoretic and immunological properties. J Biol Chem 257(10): 5449-5454.

6. Haghighi K, Kolokathis F, Pater L, Lynch RA, Asahi M, et al. (2003) Human phospholamban null results in lethal dilated cardiomyopathy revealing a critical difference between mouse and human. J Clin Invest 111(6): 869-876.

7. Acimovic I, Vilotic A, Pesl M, Lacampagne A, Dvorak P, et al. (2014) Human pluripotent stem cell-derived cardiomyocytes as research and therapeutic tools. Biomed Res Int 2014: 512831.
8. Navarrete EG, Liang P, Lan F, Sanchez-Freire V, Simmons C, et al. (2013) Screening drug-induced arrhythmia [corrected] using human induced pluripotent stem cell-derived cardiomyocytes and low-impedance microelectrode arrays. Circulation 128(1): S3-13.

9. Maillet A, Kim Tan, Xiaoran Chai, Singh N Sadananda, Ashish Mehta, et al. (2016) Modeling Doxorubicin-Induced Cardiotoxicity in Human Pluripotent Stem Cell Derived-Cardiomyocytes. Sci Rep 6: 25333.

10. Zhang J, Wilson GF, Soerens AG, Koonce CH, Yu J, et al. (2009) Functional cardiomyocytes derived from human induced pluripotent stem cells. Circ Res 104(4): e30-41.

11. Mummery CL, Zhang J, Ng ES, Elliott DA, Elefanty AG, et al. (2012) Differentiation of human embryonic stem cells and induced pluripotent stem cells to cardiomyocytes: a methods overview. Circ Res 111(3): 344-358.

12. Glass CK, Mitchell RN (2017) Winning the battle, but losing the war: mechanisms and morphology of cancer-therapy-associated cardiovascular toxicity. Cardiovasc Pathol 30: 55-63.

13.Zhao L, Zhang B (2017) Doxorubicin induces cardiotoxicity through upregulation of death receptors mediated apoptosis in cardiomyocytes. Sci Rep 7: 44735 .

14. Octavia Y, Tocchetti CG, Gabrielson KL, Janssens S, Crijns HJ, et al. (2012) Doxorubicin-induced cardiomyopathy: from molecular mechanisms to therapeutic strategies. J Mol Cell Cardiol 52(6): 1213-1225.

15. Shi Y, Moon M, Dawood S, McManus B, Liu PP (2011) Mechanisms and management of doxorubicin cardiotoxicity. Herz 36(4): 296-305.

16. Deavall DG, Martin EA, Horner JM, Roberts R (2012) Drug-induced oxidative stress and toxicity. J Toxicol 2012: 645460.

17. Khiati S, Dalla Rosa I, Sourbier C, Ma X, Rao VA, et al. (2014) Mitochondrial topoisomerase I (top1mt) is a novel limiting factor of doxorubicin cardiotoxicity. Clin Cancer Res 20(18): 4873-4881.

18. Zhang S, Liu X, Bawa-Khalfe T, Lu LS, Lyu YL, et al. (2012) Identification of the molecular basis of doxorubicin-induced cardiotoxicity. Nat Med 18(11): 1639-1642.

19. Hanna AD, Lam A, Tham S, Dulhunty AF, Beard NA (2014) Adverse effects of doxorubicin and its metabolic product on cardiac RyR2 and SERCA2A. Mol Pharmacol 86(4): 438-449.

20. Chaudhari U, Nemade H, Wagh V, Gaspar JA, Ellis JK, et al. (2016) Identification of genomic biomarkers for anthracycline-induced cardiotoxicity in human iPSC-derived cardiomyocytes: an in vitro repeated exposure toxicity approach for safety assessment. Arch Toxicol 90(11): 2763-2777.

21. Kopljar I, De Bondt A, Vinken P, Teisman A, Damiano B, et al. (2017) Chronic drug-induced effects on contractile motion properties and cardiac biomarkers in human induced pluripotent stem cell-derived cardiomyocytes. Br J Pharmacol 174(21): 3766-3779.

22. Sawaya H, Sebag IA, Plana JC, Januzzi JL, Ky B, et al. (2012) Assessment of echocardiography and biomarkers for the extended prediction of cardiotoxicity in patients treated with anthracyclines, taxanes, and trastuzumab. Circ Cardiovasc Imaging 5(5): 596-603.

23. Cardinale D, Colombo A, Torrisi R, Sandri MT, Civelli M, et al. (2010) Trastuzumab-induced cardiotoxicity: clinical and prognostic implications of troponin I evaluation. J Clin Oncol 28(25): 3910-3916.

24. Cardinale D, Sandri MT, Colombo A, Colombo N, Boeri M, et al. (2004) Prognostic value of troponin I in cardiac risk stratification of cancer patients undergoing high-dose chemotherapy. Circulation 109(22): 2749-2754.

25. Chaudhari U, Ellis JK, Wagh V, Nemade H, Hescheler J, et al. (2017) Metabolite signatures of doxorubicin induced toxicity in human induced pluripotent stem cell-derived cardiomyocytes. Amino Acids 49(12): 1955-1963. 
26. Burridge PW, Li YF, Matsa E, Wu H, Ong SG, et al. (2016) Human induced pluripotent stem cell-derived cardiomyocytes recapitulate

This work is licensed under Creative Commons Attribution 4.0 License

DOI: $10.19080 /$ CTOIJ.2018.09.555751 the predilection of breast cancer patients to doxorubicin-induced cardiotoxicity. Nat Med 22(5): 547-556.

\section{Your next submission with Juniper Publishers} will reach you the below assets

- Quality Editorial service

- Swift Peer Review

- Reprints availability

- E-prints Service

- Manuscript Podcast for convenient understanding

- Global attainment for your research

- Manuscript accessibility in different formats

( Pdf, E-pub, Full Text, Audio)

- Unceasing customer service

Track the below URL for one-step submission https://juniperpublishers.com/online-submission.php 